\title{
Influence of Laser Bonding Welding Parameters on Steel-aluminum Joint Quality

\author{
Xu Kai ${ }^{*}$, Li Guo-qiang ${ }^{1}$, Zhang Shu-quan ${ }^{2}$
}

\section{(1. Department of Mechanical Engineering,Anhui Technical College of Mechanical and Electrical Engineering, Wuhu 241000, China 2. Changzhou Institute of Technology, Changzhou 213002,China)}

Key Words: laser bonding welding; parameters; joint quality; influence

Abstract: Laser bonding welding test of the lap joint between steel and $\mathrm{Al}$ was conducted to discuss the influence of process parameters on the quality of steel-Al joint and to conduct the mechanical property analysis. The results showed that the positive weld joint of the specimen was uniformed under the conditions of $1450 \mathrm{~W}$ laser power, $25 \mathrm{~m} / \mathrm{s}$ welding speed, $+5 \mathrm{~mm}$ defocus amount and 20L/min flow rate of $\mathrm{Ar}$ as the protective gas, and continuous free from pores and cracks when glue line was added in laser welding. The shear strength and average shear force were $43.15 \mathrm{Mpa}$ and $1.13 \mathrm{KN}$, respectively. The mechanical property was slightly improved because of the glue line.

\section{Introduction}

Lightweight is an inevitable trend for the future development of automobile bodies. The steel-aluminum composite structure has a good application prospect in automobile and aviation industry etc, with the advantages of both materials. But how to get the high quality joint of dissimilar metals is the most difficult technical bottleneck for the popularization and application of steel-Al composite structure in engineering ${ }^{[1-2]}$. For example, the traditional resistance spot welding is not applicable to connect steel-Al composite automobile body, therefore new welding process must be developed to satisfy the requirements of reliable connection between steel and $\mathrm{Al}^{[3-7]}$. Laser bonding welding test of the lap joint between steel and $\mathrm{Al}$ was conducted to discuss the influence of process parameters on the quality of steel-Al joint and to conduct the mechanical property analysis.

\section{Test method}

The test facility is shown in fig. 1. The Laser used in the test is IPG YLS-4000 with rated power of $4 \mathrm{kw}$, multi-modes, focal length of $250 \mathrm{~mm}$, spot diameter at the focus of $0.42 \mathrm{~mm}$. And we also used KR60HA robot in the test. The laser head was manufactured by Beijing University of Technology. Side blowing nozzle was adopted to blow protective gas. The welder used in the test is Fronius TS-5000.

The test materials are DP590 steel and $6061 \mathrm{Al}$ alloy with size of $100 \mathrm{~mm} \times 30 \mathrm{~mm} \times 1.2 \mathrm{~mm}$ and $100 \mathrm{~mm} \times 30 \mathrm{~mm} \times 1.5 \mathrm{~mm}$, respectively. Before welding, the plates' surface was washed by acetone to remove the grease and oxidation film. Afterwards, coated glue line with thickness of $0.1 \mathrm{~mm}$ within area of $30 \mathrm{~mm}$ lap length and then imposed certain stress on the plates to make it spontaneous curing for 24 hours. During welding, the steel plate was above the Al alloy plate and Ar was used as the prtective gas. Laser bonding welding sketch is shown as fig. 2. After welding, we observed the surface shape apperance of the positive weld. The specimen was cut into standard shear specimen by linear cutting. And finally we measured the shear strength and average shear force of the specimen by microcomputre interface electronic universal testing machine.

\footnotetext{
*Brief introduction to the author: Xu Kai(1974.6-), male, master of engineering, associate professor. Main direction studied: welding technology. E-mail:ahjdxukai@126.com, Tel: 18298269028

Project: 2016 Anhui university natural science key research project, project number: KJ2016A130
} 


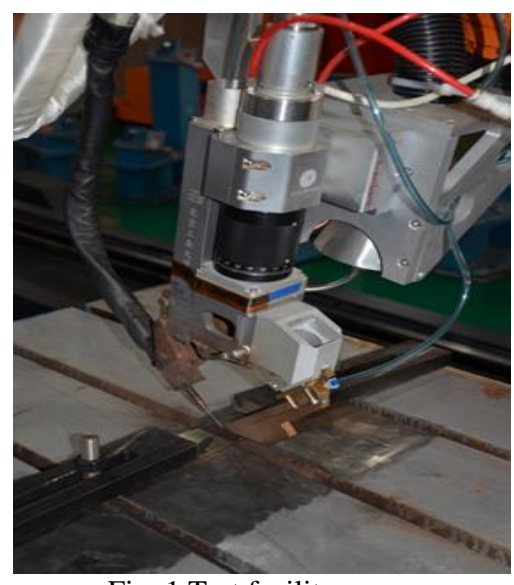

Fig. 1 Test facility

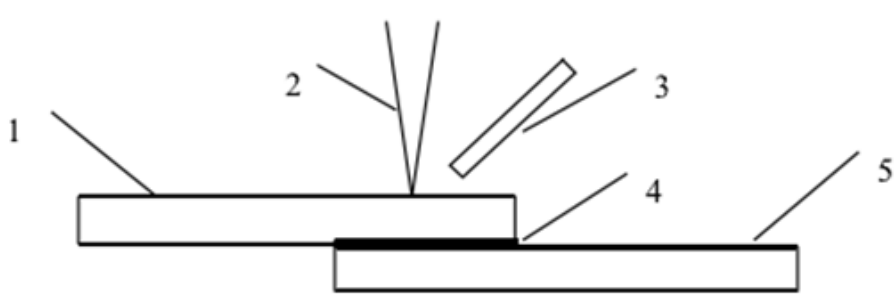

Fig. 2 Laser bonding welding sketch

1. DP590 steel 2. Laser heat source 3. Blowing side protective gas 4. Glue line 5. $6061 \mathrm{Al}$ alloy

\section{Test results and analysis}

\subsection{Influence of the laser power on the laser bonding weld joint between steel and Al}

Fig. 3 shows the positive weld shape appearance along with the change of laser power under the conditions of $25 \mathrm{~mm} / \mathrm{s}$ welding speed, $+5 \mathrm{~mm}$ defocus amount and $20 \mathrm{~L} / \mathrm{min}$ protective gas flow rate. Obviously, when the laser power increases from $1400 \mathrm{~W}$ to $1500 \mathrm{~W}$, the positive welding pool width is always within $2-2.5 \mathrm{~mm}$ with little change while the surface quality changes significantly. When the laser power is $1400 \mathrm{~W}$, the first half of the weld joint is not continuous and the molten metal splashed a lot, pits were formed in the weld zone, meanwhile, the second half weld joint is better continuous, but hump and edge subsiding occurs. When the laser power is $1450 \mathrm{~W}$, there are uniform and continuous fish scale riffles free from pores and cracks on the surface of the welding line. But when the power is increased to $1500 \mathrm{~W}$, there are obvious flaws including splashes, pits, pores, etc, on the weld joint. This indicates that low or high power will influence the surface quality of the weld joint and $1450 \mathrm{~W}$ is recommended.

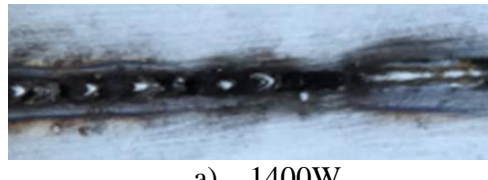

a) $1400 \mathrm{~W}$

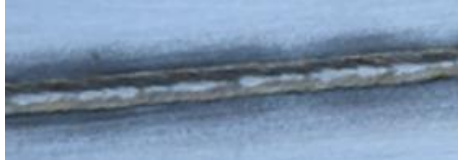

b) $1450 \mathrm{~W}$

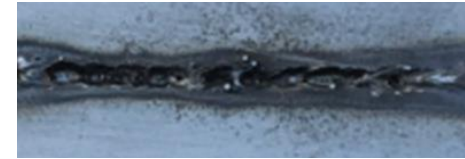

c) $1500 \mathrm{~W}$

Fig. 3 The positive weld shape appearance along with the change of laser power

\subsection{Influence of the welding speed on the laser bonding weld joint between steel and $\mathrm{Al}$}

Fig. 4 shows the positive weld shape appearance along with the change of welding speed under the conditions of $1450 \mathrm{~W}$ laser power, $+5 \mathrm{~mm}$ defocus amount and $20 \mathrm{~L} / \mathrm{min}$ protective gas flow rate. From the figure, we can notice that when the welding speed is $22 \mathrm{~mm} / \mathrm{s}$, there are pores and weld collars on the weld joint although there are already discontinuous fish scale riffles. When the speed is $25 \mathrm{~mm} / \mathrm{s}$, the weld joint is smooth and flat without obvious flaw. But when the speed is increased to $28 \mathrm{~mm} / \mathrm{s}$, there are serious splashes, pits and edge subsiding on the weld joint, which indicates that low welding speed ensures enough time for gas to escape as glue line gasifies easily during welding under slow welding speed. But when the speed is as low as $22 \mathrm{~mm} / \mathrm{s}$, the molten glue line close to the weld joint will hinder the flow of the pool, which will lead to the formation of vortex pits because of flow block and weld collars because of molten metal accumulation. When the welding speed is $28 \mathrm{~mm} / \mathrm{s}$, the splash was serious and there appeared lots of pits on the surface of the weld due to the combined action of the plasma generated from the gaseous metal and gases generated from the gaseous glue. Therefore, the welding speed recommended is $25 \mathrm{~mm} / \mathrm{s}$ 。

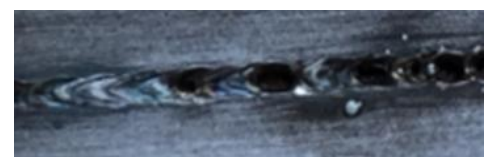

a) $22 \mathrm{~mm} / \mathrm{s}$

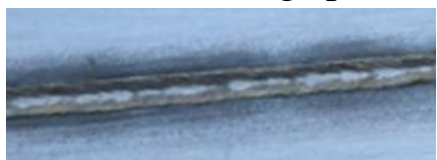

b) $25 \mathrm{~mm} / \mathrm{s}$

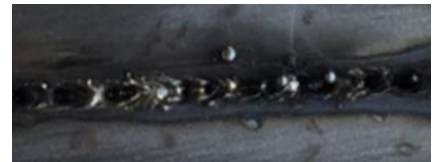

c) $28 \mathrm{~mm} / \mathrm{s}$

Fig. 4 The positive weld shape appearance along with the change of welding speed 


\subsection{Influence of the defocus amount on the laser bonding weld joint between steel and Al}

Focus position influences the size of the laser spot on the works piece surface and further changes the irradiance. Thus focus position can influence the formation of weld joint. Fig. 5 shows the positive weld shape appearance along with the change of defocus amount under the conditions of $1450 \mathrm{~W}$ laser power, $25 \mathrm{~mm} / \mathrm{s}$ welding speed and $20 \mathrm{~L} / \mathrm{min}$ protective gas flow rate. It is obvious that when the defocus amount increases from $+3 \mathrm{~mm}$ to $+5 \mathrm{~mm}$, the flaws, such as pores, pits and splashes, etc. are reducing along with the increasing defocus amount. When defocus amount is $+5 \mathrm{~mm}$, the shape of the weld joint is favorable. The reason is that the heat absorbed by the specimen surface decreases when the defocus amount increases. When there is much heat, the lower $\mathrm{Al}$ alloy plate is melted sufficiently and fully mixed with glue line and the melted steel from upper plate. Under the synergistic effect of plasma and glue line vaporization, splash happens easily, which leads to the formation of pits or pores. On the other hand, when the defocus amount is $+5 \mathrm{~mm}$, $\mathrm{Al}$ alloy melted by conductive heat of steel decreases, therefore the pool is uniform and the weld joint has good formability. So $+5 \mathrm{~mm}$ defocus amount is recommended.

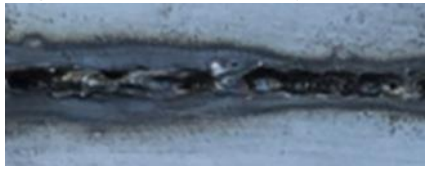

a) $+3 \mathrm{~mm}$

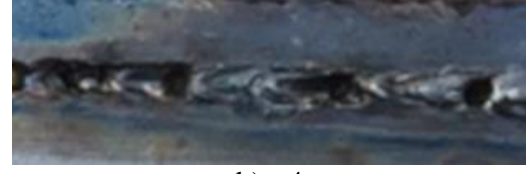

b) $+4 \mathrm{~mm}$

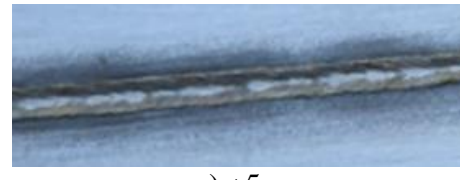

c) $+5 \mathrm{~mm}$

Fig. 5 The positive weld shape appearance along with the change of defocus amount

\subsection{Analysis of mechanical property}

Shear test of laser bonding welding plates is carried out on microcomputer interface universal electronic testing machine. The average shear force obtained is $1.13 \mathrm{KN}$ and the shear strength is $43.15 \mathrm{MPa}$, while $1.06 \mathrm{KN}$ and $42.35 \mathrm{MPa}$ for laser welding plate. So the mechanical property is slightly improved when adding glue line in laser welding.

\section{Conclusion}

(1) Under the conditions of $1450 \mathrm{~W}$ laser power, $25 \mathrm{~m} / \mathrm{s}$ welding speed, $+5 \mathrm{~mm}$ defocus amount and 20L/min flow rate of Ar as the protective gas, the positive weld joint of the specimen is uniformed and continuous free from pores and cracks when glue line is added.

(2) Addition of glue line improves the problem of heat transfer blocking in lap welding because of gap. Gap between the section metal plates decreases, depth of fusion increases and steel and $\mathrm{Al}$ are connected well.

(3) When glue line is added in laser welding, the shear strength and average shear force are $43.15 \mathrm{Mpa}$ and $1.13 \mathrm{KN}$, respectively. The mechanical property is slightly improved because of the glue line.

\section{References:}

[1]WU Jun, LI Liang. The effect of laser welding process on the welding guality of aluminum and steel[J]. Welding Technology, 2000,29(5):19-21

[2]LEI Zhen, QIN Guoliang, LIN Shangyang, et al. Fusion-brazing joining for dissimilar metals between 5A02 aluminium alloy and zinc-coated steel based on laser-MIG hybrid welding[J].Chinese Journal of Mechanical Engineering, 2009,45(3):94-98

[3]Oikawa H, Ohmiya S, Yoshimura T. Resistance spot welding of steel and aluminum sheet using insert metal sheet[J].Science and Technology of Welding and Joining,1999,4(2):80-88

[4]RanfengQiu, Hongxin Shi, Keke Zhang, et al. Interfacial characterization of joint between mild steel and aluminum alloy welded by resistance spot welding[J]. Materials Characterization, 2010,61(7):684-688

[5]QIU Ran-feng, SHI Hong-xin, ZHAN Ke-ke, et al. Research status of resistance spot welding between steel and aluminum alloy for automobile body[J]. Electric Welding Machine, 2010,40(5):150-154 
[6]YANG Yang, SHI Yan. The development of welding of dissimilar material-aluminum and steel[J]. Journal of Changchun University, 2011,21(2):21-25

[7]ZHOU Dian-wu, DAI Ting, XU Shao-hua, et al. Microstructure and properties of galvanized steel/aluminum alloy laser-adhesive hybrid bonding[J]. The Chinese Journal of Nonferrous Metals, 2014, 24(7):1736-1743 